\title{
New developments in the management of congenital Factor XIII deficiency [Corrigendum]
}

\author{
Fadoo Z, Merchant Q, Rehman KA. Journal of Blood \\ Medicine. 2013;4:65-73.
}

On page 67 the legend of Figure 1 incorrectly states "Reprinted from Song JW, Choi JR, Song KS, Rhee JH. Plasma factor XIII activity in patients with disseminated intravascular coagulation. Yonsei Med J. 2006;47(2):196-200.” The correct statement is "Reprinted from Muszbek L, Bagoly Z, Cairo A, Peyvandi F. Novel aspects of factor XIII deficiency. Curr Opin Hematol. 2011;18(5):366-372."

On page 69 the legend of Table 1 states "Reprinted from Song JW, Choi JR, Song KS, Rhee JH. Plasma factor XIII activity in patients with disseminated intravascular coagulation. Yonsei Med J. 2006;47(2):196-200.” The correct statement is "Reprinted from Kohler HP, Ichinose A, Seitz R, Ariens RA, Muszbek L; Factor XIII and fibrinogen SSC subcommittee of the ISTH. Diagnosis and classification of factor XIII deficiencies. J Thromb Haemost. 2011;9(7):1404-1406."

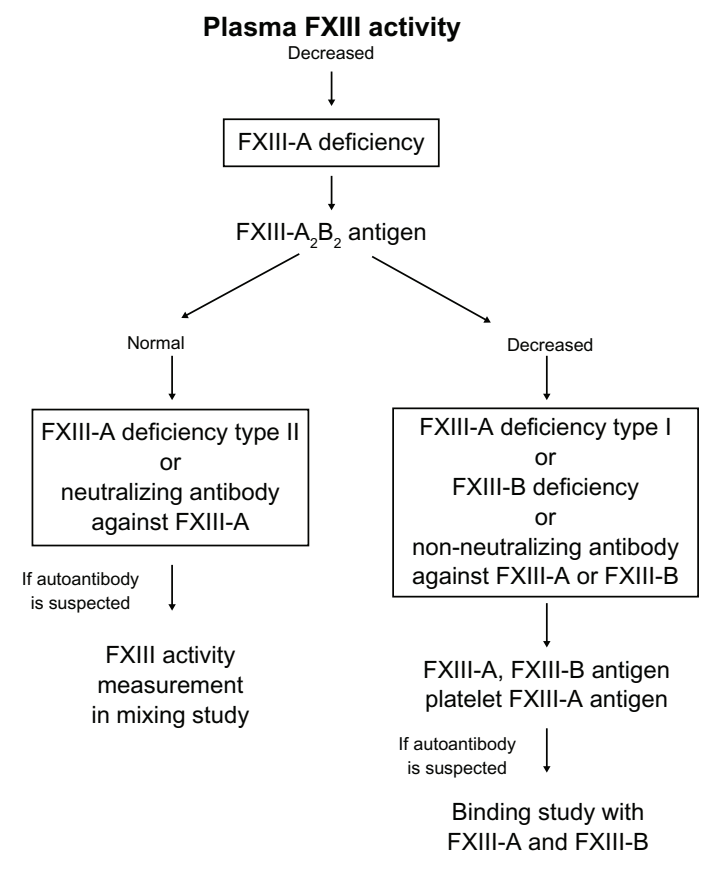

Figure I Algorithm for diagnosis and classification of Factor XIII deficiency.

Table I Laboratory Diagnosis/Classification of factor XIII deficiency

\begin{tabular}{|c|c|c|c|c|c|c|}
\hline Deficiency & $\begin{array}{l}\text { Plasma FXIII } \\
\text { activity }\end{array}$ & $\begin{array}{l}\text { Plasma FXIII- } \\
\mathrm{A}_{2} \mathrm{~B}_{2} \text { antigen }\end{array}$ & $\begin{array}{l}\text { Plasma FXIII-A } \\
\text { antigen }\end{array}$ & $\begin{array}{l}\text { Plasma FXIII-B } \\
\text { antigen }\end{array}$ & $\begin{array}{l}\text { Platelet FXIII } \\
\text { activity }\end{array}$ & $\begin{array}{l}\text { Platelet FXIII-A } \\
\text { antigen }\end{array}$ \\
\hline \multicolumn{7}{|l|}{ Inherited } \\
\hline \multicolumn{7}{|l|}{ FXIII-A deficiency } \\
\hline Type I & $\downarrow \downarrow \downarrow$ & $\downarrow \downarrow \downarrow$ & $\downarrow \downarrow \downarrow$ & $>30 \%$ & $\downarrow \downarrow \downarrow$ & $\downarrow \downarrow \downarrow$ \\
\hline Type II & $\downarrow \downarrow \downarrow$ & $\downarrow-N$ & $\downarrow-N$ & $>30 \%$ & $\downarrow \downarrow \downarrow$ & $\downarrow-N$ \\
\hline FXIII-B deficiency & $\downarrow \downarrow$ & $\downarrow \downarrow \downarrow$ & $\downarrow \downarrow$ & $\downarrow \downarrow \downarrow$ & $\mathrm{N}$ & $\mathrm{N}$ \\
\hline \multicolumn{7}{|l|}{ Autoantibody against FXIII } \\
\hline \multicolumn{7}{|l|}{ Anti-FXIII-A } \\
\hline Neutralizing & $\downarrow \downarrow \downarrow$ & $\downarrow-N$ & $\downarrow-N$ & $>30 \%$ & $\mathrm{~N}$ & $\mathrm{~N}$ \\
\hline Non-neutralizing & $\downarrow \downarrow \downarrow$ & $\downarrow \downarrow \downarrow$ & $\downarrow \downarrow \downarrow$ & $>30 \%$ & $\mathrm{~N}$ & $\mathrm{~N}$ \\
\hline Anti-FXIII-B & $\downarrow \downarrow \downarrow$ & $\downarrow \downarrow \downarrow$ & $\downarrow \downarrow \downarrow$ & $\downarrow \downarrow \downarrow$ & $\mathrm{N}$ & $\mathrm{N}$ \\
\hline Other acquired deficiencies & $\downarrow$ & $\downarrow$ & $\downarrow$ & $\downarrow-N$ & NA & NA \\
\hline
\end{tabular}

Notes: $\downarrow \downarrow \downarrow$, highly decreased activity/concentration usually below $3 \%$; $\downarrow \downarrow$, considerably decreased activity/concentration, usually $5 \%-10 \%$; $\downarrow$, slightly decreased activity, usually $20 \%-70 \%$.

Journal of Blood Medicine

\section{Publish your work in this journal}

The Journal of Blood Medicine is an international, peer-reviewed, open access, online journal publishing laboratory, experimental and clinical aspects of all topics pertaining to blood based medicine including but not limited to: Transfusion Medicine; Blood collection, Donor issues, Transmittable diseases, and Blood banking logistics; Immunohematology; Artificial and alternative blood based therapeutics; Hematology; Biotechnology/nanotechnology of blood related medicine; Legal aspects of blood medicine; Historical perspectives. The manuscript management system is completely online and includes a very quick and fair peer-review system. Visit http://www.dovepress.com/ testimonials.php to read real quotes from published authors. 